\title{
A new numerical technique for simulating the coupled seismic and electromagnetic waves in layered porous media *
}

\author{
Hengxin Ren ${ }^{1, \star}$ Qinghua Huang ${ }^{2}$ and Xiaofei Chen ${ }^{1}$ \\ ${ }^{1}$ School of Earth and Space Sciences, University of Science and Technology of China, Hefei 230026, China \\ ${ }^{2}$ Department of Geophysics, School of Earth and Space Sciences, Peking University, Beijing 100871, China
}

\begin{abstract}
Chen's technique of computing synthetic seismograms, which decomposes every vector with a set of basis of orthogonality and completeness before applying the Luco-Apsel-Chen (LAC) generalized reflection and transmission coefficients method, is confirmed to be efficient in dealing with elastic waves in multi-layered media and accurate in any frequency range. In this article, we extend Chen's technique to the computation of coupled seismic and electromagnetic (EM) waves in layered porous media. Expanding the involved mechanical and electromagnetic fields by a set of scalar and vector wave-function basis, we obtain the fundamental equations which are subsequently solved by using a recently developed version of the LAC generalized reflection and transmission coefficients method. Our approach and corresponding program is validated by reciprocity tests. We also show a numerical example of a two-layer model with an explosion source. The P-to-EM conversion waves radiated from the interface may have potential application.
\end{abstract}

Key words: synthetic seismogram; porous media; electrokinetic effect; generalized reflection and transmission coefficients

CLC number: P315.3 $\quad$ Document code: A

\section{Introduction}

Blau and Statham (1936) and Thompson (1936) were the first to report experimental results on the seismoelectric phenomenon, which means the variation of Earth resistivity with elastic deformation, called the I-effect. Ivanov (1939) discovered another seismoelectric phenomenon, called the E-effect, by measuring electric fields generated by seismic waves without applying any external voltage to the ground. In order to explain the above phenomena, Frenkel (1944) used the concept of electric double layer to develop a complete set of equations describing the electrokinetic effect in isotropic porous media. Later, Biot (1962) derived the theory of elastic waves' propagation in porous media.

Martner and Sparks (1959) reported a systematic study of electroseismic coupling using explosive sources

\footnotetext{
* Received 2 November 2009; accepted in revised form 26 November 2009; published 10 April 2010.

•Corresponding author. e-mail: renhx@ustc.edu.cn

(c) The Seismological Society of China and Springer-Verlag Berlin Heidelberg 2010
}

placed at different depths. Their results were the first to show conversions of seismic to electromagnetic (EM) energy at depth, measured at the Earth's surface with antennas. Thompson and Gist (1993) conducted field experiments, demonstrating that seismic waves can induce EM disturbances in water-saturated near-surface sediments in the Earth, and that the induced EM disturbances can be recorded at the Earth's surface. Recent field tests by Thompson et al (2007) showed the possibility of detecting hydrocarbon reservoirs at depths even up to $1000 \mathrm{~m}$ using EM-to-seismic conversion. Besides field experiments, there are also some laboratory experimental studies on seismoelectric effects. Zhu et al (2000) experimentally confirmed that the coupling between the seismic wave and EM field in the kilohertz range is electrokinetic in nature. Zhu and Toksöz (1999, 2003) performed laboratory experiments in borehole models with fractures to investigate seismoelectric conversions and confirmed EM radiation at the fractures.

Pride (1994) derived a set of macroscopic equations governing the coupled seismic and EM waves in 
fluid-saturated porous media. The properties of the coupled seismic and EM field in a fluid-saturated porous medium were discussed (Pride and Haartsen, 1996). There are two kinds of coupling: SHTE and PSVTM. In the SHTE case, SH waves generate electrical currents in the SH particle motion plane that couple to the EM-wavefield components with transverse electric (TE) polarization. In the PSVTM case, the PSV waves generate electrical currents in the PSV particle motion plane coupling to the EM-wavefield components with transverse magnetic (TM) polarization. Based on the governing equations, Haartsen and Pride (1997) and Garambois and Dietrich (2002) introduced a numerical technique modeling the coupled seismic and EM wave propagation in a fluid-saturated stratified porous medium. Haartsen and Pride (1997) used the so-called global matrix method whereas Garambois and Dietrich (2002) proposed an extension of the generalized reflection and transmission coefficients method (abbreviated as generalized $\mathrm{R} / \mathrm{T}$ coefficients method in the following context), which was originally developed by Kennett (1983). For well logging exploration case, some analytical and numerical methods were performed to describe the electroacoustic waves in borehole in porous media (Hu et al, 2003, 2007). The above algorithms were implemented in the frequency domain. There are also a few numerical simulations in the time domain, e.g., Han and Wang (2001) provided a finite element algorithm of modeling the seismo-electromagnetic field induced by SH waves, Haines and Pride (2006) presented a finite difference algorithm of modeling seismoelectric phenomena and provided a 2D implementation of this algorithm. Guan and $\mathrm{Hu}$ (2008) provided a finite-difference time-domain algorithm modeling the electroseismic logging in fluid-saturated porous media.

As mentioned above, the model of Pride (1994) has been widely adopted to investigate the coupling of seismic and EM waves in porous media. However, it should be also mentioned that Revil and Linde (2006) developed recently a new and alternative theory of the electrokinetic behavior of consolidated rocks at low frequencies. While this theory was first developed to study the electrokinetic properties of clay-rocks (Revil et al, 2003; Revil and Leroy, 2004), this model also works well for high porosity reservoir rocks, soils, and packs of glass beads (Bolève et al, 2007a, b).

Chen (1993) has developed the generalized R/T coefficients method, which was originally introduced by Luco and Apsel (1983), to the study of computing normal modes for multilayered half-space. This developed method was adopted by Park (1996) and referred as LAC R/T scheme in the study of Martin and Thomson (1997) who compared this scheme with Kennett R/T scheme (Kennett, 1983) and affirmed the priority of LAC R/T scheme in numerical calculation stability. Chen (1999) developed a method to calculate synthetic seismograms for layered media, decomposing every vector by using a set of basis of orthogonality and completeness before applying the LAC generalized R/T coefficients method. Chen (2007) extended the LAC generalized $\mathrm{R} / \mathrm{T}$ coefficients method to the study of generation and propagation of seismic $\mathrm{SH}$ waves in multi-layered media with irregular interfaces. Quite recently, Ge and Chen (2008) further developed the LAC generalized $\mathrm{R} / \mathrm{T}$ coefficients method by introducing a more straightforward evaluation of the generalized $\mathrm{R} / \mathrm{T}$ coefficients, which makes this approach more efficient.

In the present study, we extend the above method of calculating seismograms (Chen, 1993, 1999, 2007; Ge and Chen, 2008) to the numerical simulation of the coupled seismic and EM waves in layered porous media. We focus on the saturated porous case and adopt the model described by the macroscopic dynamic governing equations of Pride (1994). After decomposing each vector in a cylindrical coordinate system, we obtain the fundamental equation corresponding to both PSVTM and SHTE models, as well as two additional equations related to the vertical component of EM waves. Then we use the improved LAC generalized $\mathrm{R} / \mathrm{T}$ coefficients method, in which the straightforward evaluation of the generalized $\mathrm{R} / \mathrm{T}$ coefficients is adopted, to solve the fundamental equation. We show that the general reciprocity theorem of Green's tensor is satisfied for our program. Then we apply our new technique to a two-layer porous media with an explosion source. This work may provide an effective way to investigate seismo-electromagnetic effects in porous media, which is useful for monitoring the pumping of oil and gas reservoirs (Thompson and Gist, 1993; Thompson et al, 2007; Pride and Haartsen, 1996; Revil et al, 2003) and the possible EM disturbances associated with earthquakes and volcano eruptions (Park et al, 1993; Johnston, 1997; Huang, 2002; Huang and Liu, 2006; Nagao et al, 2002). 


\section{Methodology}

\subsection{Governing equations}

Assuming a time dependence of $\mathrm{e}^{-\mathrm{i} \omega t}$, Pride (1994) derived the macroscopic governing equations in frequency domain, which control the propagation of coupled seismic and EM waves in porous media. We adopted the following governing equations in our numerical simulations based on the model of Pride (1994) and Haartsen and Pride (1997), while the first two equations were rewritten after taking into account the problem to be dealt with in this paper.

$$
\begin{gathered}
-\rho \omega^{2} \boldsymbol{u}-\rho_{\mathrm{f}} \omega^{2} \boldsymbol{w}=\left(K_{G}+\frac{4}{3} G\right) \nabla(\nabla \cdot \boldsymbol{u})- \\
G \nabla \times \nabla \times \boldsymbol{u}+C \nabla(\nabla \cdot \boldsymbol{w})+\boldsymbol{F}, \\
\boldsymbol{\tau}=\left[\left(K_{G}-\frac{2 G}{3}\right) \nabla \cdot \boldsymbol{u}+C \nabla \cdot \boldsymbol{w}\right] \hat{z}+ \\
G\left[2 \frac{\partial \boldsymbol{u}}{\partial z}+\hat{z} \times(\nabla \times \boldsymbol{u})\right] \\
-P=C \nabla \cdot \mathbf{u}+M \nabla \cdot \mathbf{w} \\
-\mathrm{i} \omega \boldsymbol{w}=\frac{\kappa}{\eta}\left[-\nabla P+\rho_{\mathrm{f}} \omega^{2} \boldsymbol{u}+f\right]+L \boldsymbol{E} \\
\boldsymbol{J}=L\left[-\nabla P+\rho_{\mathrm{f}} \omega^{2} \boldsymbol{u}+f\right]+\sigma \boldsymbol{E} \\
\nabla \times \boldsymbol{E}=\mathrm{i} \omega \mu \boldsymbol{H}-\boldsymbol{M} \\
\nabla \times \boldsymbol{H}=-\mathrm{i} \omega \varepsilon \boldsymbol{E}+\boldsymbol{J}+C
\end{gathered}
$$

where $\boldsymbol{E}$ is the electric field, $\boldsymbol{H}$ the magnetic field, $\boldsymbol{J}$ the electric current, $\tau$ the traction acting on the horizontal plane at depth $z, P$ the pore-fluid pressure, $\boldsymbol{u}$ the average displacement of solid, $\boldsymbol{w}$ the average relative fluid-solid displacement multiplied by porosity, $\rho$ the bulk density, $\rho_{\mathrm{f}}$ the fluid density, $\varepsilon$ the electrical permittivity, $\mu$ the magnetic permeability, $\omega$ the radial frequency, $F$ and $f$ the applied body-force densities acting on the bulk material and fluid phases respectively (here a minus sign is added to $F$ to let $F$ and $f$ have the consistent physical meanings), $I$ the identity matrix, $C$ the applied electric current source, $M$ the magnetic current sources, $L$ the electrokinetic coupling coefficient, $\kappa$ the dynamic permeability, $\eta$ the fluid viscosity, $K_{\mathrm{G}}$ the Gassmann's bulk modulus, $G$ the shear modulus of the solid frame, $C$ and $M$ related to the bulk modulus of the solid and fluid phases and the bulk modulus of the drained frame of the solid phase.

\subsection{Layered media and general solutions}

The model concerned in this paper is a layered porous media with $N$ homogeneous layers over a half-space. The $j$-th layer is bounded by horizontal flat interfaces, $z=z^{(j-1)}$ and $z=z^{(j)}$. The top layer can either be a half-space or have a free surface with $z=z^{(0)}$. The source is placed in the $s$-th layer, which is not a half-space, i.e., $s<N+1$ and $s>1$ while the top layer is a half-space.

The governing equations for the seismo-electromagnetic problem, as shown in equations (1) to (7), involve both scalar and vector functions. These functions are expanded by using a set of scalar and vector wave-function basis, whose detailed expressions are described in Appendix A. Thus, we obtained two sets of linear ordinary differential equations corresponding to the SHTE model and PSVTM model respectively. Both have the following form

$$
\begin{gathered}
\frac{\partial}{\partial z} \boldsymbol{y}^{(j)}(z)=\boldsymbol{A}^{(j)} \boldsymbol{y}^{(j)}(z)+\boldsymbol{\delta}_{j, s} \boldsymbol{F}(z), \\
\text { for } \quad z^{(j-1)}<z<z^{(j)}
\end{gathered}
$$

where $j=1,2, \cdots, N$, and $\delta_{j, s}$ is Kronecker delta function.

The $y$ vectors of the two models are written as

$$
\begin{aligned}
& \boldsymbol{y}^{\mathrm{SHTE}}=\left[\begin{array}{llll}
u_{T, m} & \tau_{T, m} & H_{S, m} & E_{T, m}
\end{array}\right]^{\mathrm{T}}, \\
& \boldsymbol{y}^{\mathrm{PSVTM}}= \\
& {\left[\begin{array}{llllllll}
u_{S, m} & u_{R, m} & w_{R, m} & \tau_{S, m} & \tau_{R, m} & \tilde{P} & H_{T, m} & E_{S, m}
\end{array}\right]^{\mathrm{T}},}
\end{aligned}
$$

where $u_{T, m}, u_{S, m}, u_{R, m}, w_{R, m}, \tau_{T, m}, \tau_{S, m}, \tau_{R, m}, H_{T, m}, H_{S, m}$, $H_{R, m}$ [equation (11)], $E_{T, m}, E_{S, m}, E_{R, m}$ [equation (12)], and $\tilde{P}$ are the corresponding expansion coefficients of unknown vector functions $\boldsymbol{u}, \boldsymbol{w}, \boldsymbol{\tau}, \boldsymbol{H}, \boldsymbol{E}$ and the unknown pore-fluid pressure $P$, respectively. The explicit expressions of $\boldsymbol{A}$ and $\boldsymbol{F}$ are given in Appendix B.

Two extra equations corresponding to the vertical components of EM waves are

$$
\begin{gathered}
\mathrm{i} \mu \omega H_{R, m}=-k E_{T, m}+M_{R, m} \quad \text { (for SHTE model); } \\
k E_{R, m}=-\frac{\partial}{\partial z} E_{S, m}-\mathrm{i} \mu \omega H_{T, m}+M_{T, m} \\
\text { (for PSVTM model). }
\end{gathered}
$$

Once we have known $H_{T, m} \quad E_{T, m}$ and $\frac{\partial}{\partial z} E_{S, m}$, we can calculate the vertical components of EM waves, $E_{R, m}$ and $H_{R, m}$.

The general solution of the equation (8) can be written as 


$$
\begin{gathered}
\boldsymbol{y}^{(j)}(z)=\boldsymbol{\Theta}^{(j)} \boldsymbol{\Lambda}^{(j)}(z) \cdot \\
\left\{\boldsymbol{a}^{(j)}+\delta_{j, s} \int^{z}\left[\boldsymbol{\Lambda}^{(s)}(\xi)\right]^{-1}\left(\boldsymbol{\Theta}^{(s)}\right)^{-1} \boldsymbol{F}(\xi) \mathrm{d} \xi\right\}
\end{gathered}
$$

where $j=1,2, \cdots, N+1$. The wave amplitude vector $\boldsymbol{a}^{(j)}$ is an unknown constant vector to be determined by boundary conditions. $\boldsymbol{\Theta}^{(j)}$ and $\boldsymbol{\Lambda}^{(j)}(z)$ are the matrices related to the eigen-vectors and eigen-values of $\boldsymbol{A}^{(j)}$ as follows

$$
\begin{gathered}
\boldsymbol{A}^{(j)} \boldsymbol{e}_{v}=\gamma_{v} \boldsymbol{e}_{v}, \quad \text { for } \quad v=1,2, \cdots, n, \\
\boldsymbol{\Theta}^{(j)}=\left[\begin{array}{llll}
\boldsymbol{e}_{1} & \boldsymbol{e}_{2} & \cdots & \boldsymbol{e}_{n}
\end{array}\right], \\
\boldsymbol{\Lambda}^{(j)}(z)=\operatorname{diag}\left\{\mathrm{e}^{-\gamma_{1}\left[z-z^{(j-1)}\right]}, \mathrm{e}^{-\gamma_{2}\left[z-z^{(j-1)}\right]}, \cdots, \mathrm{e}^{-\gamma_{m}\left[z-z^{(j-1)}\right]},\right. \\
\left.\mathrm{e}^{-\gamma_{1}\left[z^{(j)}-z\right]}, \mathrm{e}^{-\gamma_{2}\left[z^{(j)}-z\right]} \cdots, \mathrm{e}^{-\gamma_{m}\left[z^{(j)}-z\right]}\right\} .
\end{gathered}
$$

For SHTE model, $n=4$, we get two pairs of eigen-values, $\pm \gamma_{i}$ with $\operatorname{Re}\left\{\gamma_{i}\right\}>0$ for $i=1$, 2; For PSVTM model, $n=8$, we get four pairs of eigen-values, $\pm \gamma_{i}$ with $\operatorname{Re}\left\{\gamma_{i}\right\}>0$ for $i=1,2,3,4$.

It should be stressed that a slight modification has been applied to solution (13), thus the diagonal matrix $\boldsymbol{\Lambda}^{(j)}(z)$ contains exponential decay terms only.

\subsection{LAC generalized $R / T$ coefficients method}

The general solution can be written in matrix form as follows

$$
\begin{aligned}
& \boldsymbol{y}^{(j)}(z)=\left[\begin{array}{ll}
\boldsymbol{\Theta}_{11}^{(j)} & \boldsymbol{\Theta}_{12}^{(j)} \\
\boldsymbol{\Theta}_{21}^{(j)} & \boldsymbol{\Theta}_{22}^{(j)}
\end{array}\right]\left[\begin{array}{cc}
\boldsymbol{\Lambda}_{\mathrm{d}}^{(j)}(z) & 0 \\
0 & \boldsymbol{\Lambda}_{\mathrm{u}}^{(j)}(z)
\end{array}\right] . \\
& {\left[\begin{array}{l}
\boldsymbol{a}_{\mathrm{d}}^{(j)}+\delta_{j, s} \boldsymbol{b}_{\mathrm{d}}(z) \\
\boldsymbol{a}_{\mathrm{u}}^{(j)}+\delta_{j, s} \boldsymbol{b}_{\mathrm{u}}(z)
\end{array}\right] }
\end{aligned}
$$

where $\boldsymbol{\Theta}_{11}^{(j)}, \boldsymbol{\Theta}_{12}^{(j)}, \boldsymbol{\Theta}_{21}^{(j)}$ and $\boldsymbol{\Theta}_{22}^{(j)}$ are four sub-matrices of $\boldsymbol{\Theta}^{(j)}, \boldsymbol{\Lambda}_{\mathrm{d}}^{(j)}(z)$ and $\boldsymbol{\Lambda}_{\mathrm{u}}^{(j)}(z)$ represent the down-going and up-going waves respectively, while $\boldsymbol{a}_{d}^{(j)}$ and $\boldsymbol{a}_{u}^{(j)}$ are the corresponding amplitudes of these down-going and up-going waves, and $\boldsymbol{b}_{\mathrm{d}}(z)$ and $\boldsymbol{b}_{\mathrm{u}}(z)$ relate to the source as follows

$$
\begin{gathered}
\boldsymbol{b}_{\mathrm{d}}(z)=\int_{z^{(s-1)}}^{z}\left[\boldsymbol{\Lambda}_{d}^{(s)}(\xi)\right]^{-1}\left\{\left[\left(\boldsymbol{\Theta}^{(s)}\right)^{-1}\right]_{11} \boldsymbol{F}_{1}(\xi)+\right. \\
\left.\left[\left(\boldsymbol{\Theta}^{(s)}\right)^{-1}\right]_{12} \boldsymbol{F}_{2}(\xi)\right\} \mathrm{d} \xi, \\
\boldsymbol{b}_{\mathrm{u}}(z)=\int_{z^{(s)}}^{z}\left[\boldsymbol{\Lambda}_{u}^{(s)}(\xi)\right]^{-1}\left\{\left[\left(\boldsymbol{\Theta}^{(s)}\right)^{-1}\right]_{21} \boldsymbol{F}_{1}(\xi)+\right. \\
\left.\left[\left(\boldsymbol{\Theta}^{(s)}\right)^{-1}\right]_{22} \boldsymbol{F}_{2}(\xi)\right\} \mathrm{d} \xi .
\end{gathered}
$$

where $\left[\left(\Theta^{(s)}\right)^{-1}\right]_{11},\left[\left(\Theta^{(s)}\right)^{-1}\right]_{12},\left[\left(\Theta^{(s)}\right)^{-1}\right]_{21}$, and $\left[\left(\Theta^{(s)}\right)^{-1}\right]_{22}$ are four sub-matrices of $\left(\Theta^{(s)}\right)^{-1}$.

The case of $j=1$ of equation (18) is correct when the top layer has a free surface. However, if the top layer is a half-space, there are no down-going waves, thus

$$
\boldsymbol{a}_{\mathrm{d}}^{(1)}=0, \quad \text { and } \quad \boldsymbol{y}^{(1)}(z)=\left[\begin{array}{c}
\boldsymbol{\Theta}_{12}^{(1)} \boldsymbol{\Lambda}_{\mathrm{u}}^{(1)}(z) \boldsymbol{a}_{\mathrm{u}}^{(1)} \\
\boldsymbol{\Theta}_{22}^{(1)} \boldsymbol{\Lambda}_{\mathrm{u}}^{(1)}(z) \boldsymbol{a}_{\mathrm{u}}^{(1)}
\end{array}\right] \text {. }
$$

In the bottom half-space, there are no up-going waves, thus

$$
\begin{gathered}
\boldsymbol{a}_{\mathrm{u}}^{(N+1)}=0, \text { and } \\
\boldsymbol{y}^{(N+1)}(z)=\left[\begin{array}{l}
\boldsymbol{\Theta}_{11}^{(N+1)} \boldsymbol{\Lambda}_{\mathrm{d}}^{(N+1)}(z) \boldsymbol{a}_{\mathrm{d}}^{(N+1)} \\
\boldsymbol{\Theta}_{21}^{(N+1)} \boldsymbol{\Lambda}_{\mathrm{d}}^{(N+1)}(z) \boldsymbol{a}_{\mathrm{d}}^{(N+1)}
\end{array}\right] .
\end{gathered}
$$

Now, in order to determine the unknown wave-amplitude vector $\boldsymbol{a}^{(j)}$, we introduce the LAC generalized $\mathrm{R} / \mathrm{T}$ coefficients method.

\subsubsection{Straightforward evaluation of generalized $R / T$ coefficients}

The generalized $\mathrm{R} / \mathrm{T}$ coefficients are defined as follows

When the top layer is a half-space, $\boldsymbol{R}_{\mathrm{ud}}^{(0)}=0$; and

$$
\boldsymbol{a}_{\mathrm{d}}^{(1)}=\boldsymbol{R}_{\mathrm{ud}}^{(0)}\left[\boldsymbol{a}_{\mathrm{u}}^{(1)}+\delta_{1, s} \boldsymbol{b}_{\mathrm{u}}\left(z^{(0)}\right)\right],
$$

when the top layer has a free surface; and

$$
\begin{gathered}
\left\{\begin{array}{c}
\boldsymbol{a}_{\mathrm{u}}^{(j)}=\boldsymbol{T}_{\mathrm{u}}^{(j)}\left[\boldsymbol{a}_{\mathrm{u}}^{(j+1)}+\delta_{j+1, s} \boldsymbol{b}_{\mathrm{u}}\left(z^{(j)}\right)\right] \\
\boldsymbol{a}_{\mathrm{d}}^{(j+1)}=\boldsymbol{T}_{\mathrm{ud}}^{(j)}\left[\boldsymbol{a}_{\mathrm{u}}^{(j+1)}+\delta_{j+1, s} \boldsymbol{b}_{\mathrm{u}}\left(z^{(j)}\right)\right]
\end{array}\right. \\
(j=1,2, \cdots, s-1), \\
\left\{\begin{array}{c}
\boldsymbol{a}_{\mathrm{u}}^{(j)}=\boldsymbol{T}_{\mathrm{d}}^{(j)}\left[\boldsymbol{a}_{\mathrm{d}}^{(j+1)}+\delta_{j, s} \boldsymbol{b}_{\mathrm{d}}\left(z^{(j)}\right)\right] \\
\boldsymbol{a}_{\mathrm{u}}^{(j+1)}=\boldsymbol{T}_{\mathrm{du}}^{(j)}\left[\boldsymbol{a}_{\mathrm{d}}^{(j+1)}+\delta_{j+1, s} \boldsymbol{b}_{\mathrm{d}}\left(z^{(j)}\right)\right] \\
(j=s, s+1, \cdots, N) .
\end{array}\right.
\end{gathered}
$$

According to the continuity condition at the interface, we obtain

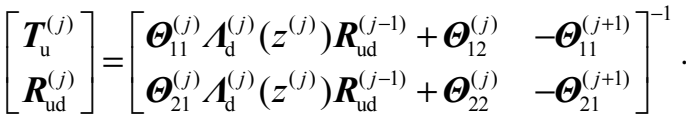

$$
\begin{aligned}
& {\left[\begin{array}{l}
\boldsymbol{\Theta}_{12}^{(j+1)} \\
\boldsymbol{\Theta}_{22}^{(j+1)}
\end{array}\right] \boldsymbol{\Lambda}_{u}^{(j+1)}\left(z^{(j)}\right),(j=1,2, \cdots, s-1) ;} \\
& {\left[\begin{array}{l}
\boldsymbol{T}_{\mathrm{d}}^{(j)} \\
\boldsymbol{R}_{\mathrm{du}}^{(j)}
\end{array}\right]=\left[\begin{array}{ll}
\boldsymbol{\Theta}_{11}^{(j+1)}+\boldsymbol{\Theta}_{12}^{(j+1)} \boldsymbol{\Lambda}_{\mathrm{u}}^{(j+1)}\left(z^{(j)}\right) \boldsymbol{R}_{\mathrm{du}}^{(j+1)} & -\boldsymbol{\Theta}_{12}^{(j)} \\
\boldsymbol{\Theta}_{21}^{(j+1)}+\boldsymbol{\Theta}_{22}^{(j+1)} \boldsymbol{\Lambda}_{\mathrm{u}}^{(j+1)}\left(z^{(j)}\right) \boldsymbol{R}_{\mathrm{du}}^{(j+1)} & -\boldsymbol{\Theta}_{22}^{(j)}
\end{array}\right]^{-1} .} \\
& {\left[\begin{array}{c}
\boldsymbol{\Theta}_{11}^{(j)} \\
\boldsymbol{\Theta}_{21}^{(j)}
\end{array}\right] \boldsymbol{\Lambda}_{\mathrm{d}}^{(j)}\left(z^{(j)}\right),(j=s, s+1, \cdots, N) .}
\end{aligned}
$$


Obviously, in order to calculate the above coefficients, we have to get the values of $\boldsymbol{R}_{\mathrm{ud}}^{(0)}$ and $\boldsymbol{R}_{\mathrm{du}}^{(N+1)}$, whose explicit expressions are obtained through two boundary conditions. The detailed equations are listed in Appendix C.

\subsubsection{Determination of unknown wave-amplitude vector}

Since we have now gotten the generalized R/T coefficients, we can determine the unknown wave-amplitude vector $\boldsymbol{a}^{(j)}$, which is divided into two sub-vectors $\boldsymbol{a}_{\mathrm{d}}^{(j)}$ and $\boldsymbol{a}_{\mathrm{u}}^{(j)}$ written as follows,

$$
\left\{\begin{array}{l}
\boldsymbol{a}_{\mathrm{d}}^{(s)}+\boldsymbol{s}_{\mathrm{d}}=\left[\boldsymbol{I}-\boldsymbol{R}_{\mathrm{ud}}^{(s-1)} \boldsymbol{R}_{\mathrm{du}}^{(s)}\right]^{-1}\left[\boldsymbol{s}_{\mathrm{d}}+\boldsymbol{R}_{\mathrm{ud}}^{(s-1)} \boldsymbol{s}_{\mathrm{u}}\right], \\
\boldsymbol{a}_{\mathrm{u}}^{(s)}+\boldsymbol{s}_{\mathrm{u}}=\left[\boldsymbol{I}-\boldsymbol{R}_{\mathrm{du}}^{(s)} \boldsymbol{R}_{\mathrm{ud}}^{(s-1)}\right]^{-1}\left[\boldsymbol{s}_{\mathrm{u}}+\boldsymbol{R}_{\mathrm{du}}^{(s)} \boldsymbol{s}_{\mathrm{d}}\right]
\end{array},\right.
$$

and

$$
\begin{aligned}
& \left\{\begin{array}{l}
\boldsymbol{a}_{\mathrm{u}}^{(j)}=\boldsymbol{T}_{\mathrm{u}}^{(j)} \boldsymbol{T}_{\mathrm{u}}^{(j+1)} \cdots \boldsymbol{T}_{\mathrm{u}}^{(s-1)}\left[\boldsymbol{a}_{\mathrm{u}}^{(s)}+\boldsymbol{s}_{\mathrm{u}}\right] \\
\boldsymbol{a}_{\mathrm{d}}^{(j)}=\boldsymbol{T}_{\mathrm{ud}}^{(j-1)} \boldsymbol{a}_{\mathrm{u}}^{(j)}
\end{array}\right. \\
& (j=1,2, \cdots, s-1), \\
& \left\{\begin{array}{l}
\boldsymbol{a}_{\mathrm{d}}^{(j)}=\boldsymbol{T}_{\mathrm{d}}^{(j-1)} \boldsymbol{T}_{\mathrm{d}}^{(j-2)} \cdots \boldsymbol{T}_{\mathrm{d}}^{(s)}\left[\boldsymbol{a}_{\mathrm{d}}^{(s)}+\boldsymbol{s}_{\mathrm{d}}\right] \\
\boldsymbol{a}_{\mathrm{u}}^{(j)}=\boldsymbol{R}_{\mathrm{du}}^{(j)} \boldsymbol{a}_{\mathrm{d}}^{(j)}
\end{array}\right. \\
& (j=s+1, s+2, \cdots, N+1) ;
\end{aligned}
$$

where $\boldsymbol{s}_{\mathrm{d}}=\boldsymbol{b}_{\mathrm{d}}\left(z^{(s)}\right)$ and $\boldsymbol{s}_{\mathrm{u}}=\boldsymbol{b}_{\mathrm{u}}\left(z^{(s-1)}\right)$ are source terms corresponding to the down-going and up-going waves respectively.

\subsubsection{Vertical components of EM waves}

According to equations (11) and (12), the vertical components of EM waves are relative to the horizontal components of EM waves and magnetic-current source. We can get $E_{T, m}$ from equation (17) for SHTE model, so we can compute $H_{R, m}$ according to equation (11). In order to get $E_{R, m}$ according to equation (12), we have to know $H_{T, m}$ which is given by equation (17) for PSVTM model and $\frac{\partial}{\partial z} E_{S, m}$ which can be obtained from the derivative of equation (17) written as

$$
\begin{gathered}
\frac{\partial}{\partial z} \boldsymbol{y}^{(j)}(z)=\left[\begin{array}{ll}
\boldsymbol{\Theta}_{11}^{(j)} & \boldsymbol{\Theta}_{12}^{(j)} \\
\boldsymbol{\Theta}_{21}^{(j)} & \boldsymbol{\Theta}_{22}^{(j)}
\end{array}\right]\left[\begin{array}{cc}
\boldsymbol{\Psi} & 0 \\
0 & -\boldsymbol{\Psi}
\end{array}\right] . \\
{\left[\begin{array}{cc}
\boldsymbol{\Lambda}_{\mathrm{d}}^{(j)}(z) & 0 \\
0 & \boldsymbol{\Lambda}_{\mathrm{u}}^{(j)}(z)
\end{array}\right]\left[\begin{array}{l}
\boldsymbol{a}_{\mathrm{d}}^{(j)} \\
\boldsymbol{a}_{\mathrm{u}}^{(j)}
\end{array}\right]}
\end{gathered}
$$

where $\boldsymbol{\Psi}=\operatorname{diag}\left\{\begin{array}{llll}-\gamma_{1}^{(j)} & -\gamma_{2}^{(j)} & -\gamma_{3}^{(j)} & -\gamma_{4}^{(j)}\end{array}\right\} . \quad$ The vectors and matrices are those for PSVTM model, thus $\frac{\partial}{\partial z} E_{S, m}^{(j)}$ is the eighth element of $\frac{\partial}{\partial z} \boldsymbol{y}^{(j)}(z)$ given by equation (30).

Having derived the components of every field vector in the coordinates system with vector basis given by equation (A-3), we can now get the components of every vector in cylindrical coordinates system according to equation (A-4).

\subsubsection{Back in the space-time domain}

Having obtained the displacement-stress-EM wavefields in the frequency-wavenumber domain, we now consider the transformation back to the space-time domain, which can be performed by discrete wave number (DWN) method. Bouchon (2003) reviewed the DWN method, introduced by Bouchon and Aki (1977) and Bouchon (1981), which uses a spatial periodicity of sources to discretize the radiated wave fields and relies on the Fourier transform in the complex frequency domain.

The integral over the horizontal wave number $k$ can be discretely evaluated by introducing an infinite set of concentric rings of secondary sources distributed periodically with an equal radial interval, which is required to be large enough to make sure that the waves generated by the additional sources do not enter the time window (Bouchon, 1981, 2003). In order to remove the singularities from the real $k$ axis, a small constant imaginary part is added to the real frequency. The effect of the imaginary part frequency can be removed from the final time domain in the process of inverse Fourier transform (Bouchon and Aki, 1977).

\section{Reciprocity test and one numerical example}

In order to test our numerical simulation technique and our program, we performed the reciprocity test, which is a useful method for checking of numerical simulations program. The reciprocity theorem can be written as

$$
\boldsymbol{G}_{j}^{i}\left(\boldsymbol{x}_{r}, t ; \boldsymbol{x}_{s}, t_{0}\right)=\boldsymbol{G}_{i}^{j}\left(\boldsymbol{x}_{s}, t ; \boldsymbol{x}_{r}, t_{0}\right),
$$

where $\boldsymbol{G}_{j}^{i}\left(\boldsymbol{x}_{r}, t ; \boldsymbol{x}_{s}, t_{0}\right)(i=1,3 ; j=1,3)$ represents the Green's tensor along direction $i$, recorded at $\left(\boldsymbol{x}_{r}, t\right)$ which are generated by a point source oriented in direction $j$, located at $\boldsymbol{x}_{s}$ and acting at time $t_{0}$.

For coupled seismic and EM waves in porous media, reciprocity can be verified for (1) seismic waves generated by seismic point sources, (2) electric waves 
generated by current sources, and (3) elastic waves generated by current sources and electric waves generated by seismic point sources (Pride and Haartsen, 1996). We tested all the above kinds of reciprocity. However, for saving space, we only show the results of the third reciprocity test, which is the most interesting case.

This reciprocity test is carried out with a threelayer model, which has a 100-m-thick porous layer $\left(l_{2}\right)$ sandwiched between two identical half-spaces $\left(l_{1}\right)$. The properties of layers are listed in Table 1. Two reciprocal source-receiver configurations are used. One is the down-going configuration and the other is the up-going configuration. In the down-going configuration, the seismic source is located in the top half-space $100 \mathrm{~m}$ above first interface while the receiver is located at the bottom half-space $100 \mathrm{~m}$ below the second interface with lateral offsets of $x=50 \mathrm{~m}$ and $y=100 \mathrm{~m}$. Thus, in the up-going configuration, the receiver is located in the top half-space while the source is located at the bottom half-space. The seismic source is a point single force $\boldsymbol{F}$ whose magnitude is $1 \mathrm{~N}$. The current density source $D$ is created by a 1-m-long dipole antenna carrying a 1-A current. The sources are chosen to be oriented in $x, y$, or $z$ directions. The applied source time function is a zero-phase Ricker wavelet with a dominant frequency of $100 \mathrm{~Hz}$.

Table 1 Properties of the homogeneous layers used in the numerical calculation

\begin{tabular}{lllll}
\hline \multirow{2}{*}{\multicolumn{1}{c}{ Properties }} & \multicolumn{4}{c}{ layre } \\
\cline { 2 - 5 } & \multicolumn{1}{c}{$l_{1}$} & \multicolumn{1}{c}{$l_{2}$} & sand & sandstone \\
\hline Porosity $\phi$ & $15 \%$ & $30 \%$ & $30 \%$ & $20 \%$ \\
DC permeability $k_{0} / \mathrm{m}^{2}$ & $10^{-12}$ & $10^{-11}$ & $10^{-11}$ & $10^{-12}$ \\
Solid bulk modulus $k_{\mathrm{s}} / \mathrm{GPa}$ & 36 & 36 & 35 & 36 \\
Fluid bulk modulus $k_{\mathrm{f}} / \mathrm{GPa}$ & 2.2 & 2.2 & 2.2 & 2.2 \\
Frame bulk modulus $k_{\mathrm{fr}} / \mathrm{GPa}$ & 9.0 & 7.0 & 0.4 & 5.0 \\
Frame shear modulus $G_{\mathrm{fr}} / \mathrm{GPa}$ & 7.0 & 5.0 & 0.5 & 7.0 \\
Fluid viscosity $\eta / \mathrm{Pa} \cdot \mathrm{s}$ & $10^{-3}$ & $10^{-3}$ & $10^{-3}$ & $10^{-3}$ \\
Solid density $\rho_{\mathrm{s}} / \mathrm{kg} \cdot \mathrm{m}^{-3}$ & 2700 & 2700 & 2600 & 2700 \\
Fluid density $\rho_{\mathrm{f}} / \mathrm{kg} \cdot \mathrm{m}^{-3}$ & $10^{3}$ & $10^{3}$ & $10^{3}$ & $10^{3}$ \\
Salinity $C_{0} / \mathrm{mol}^{-1} \mathrm{~L}^{-1}$ & $10^{-2}$ & $10^{-4}$ & $10^{-3}$ & $10^{-3}$ \\
Temperature $T / \mathrm{K}$ & 298 & 298 & 298 & 298 \\
Fluid Permittivity $\kappa_{\mathrm{f}}$ & 80 & 80 & 80 & 80 \\
Solid Permittivity $\kappa_{\mathrm{s}}$ & 4 & 4 & 4 & 4 \\
Tortuosity $\alpha_{\infty}$ & 3 & 3 & 3 & 3 \\
\hline
\end{tabular}

As shown in Figure 1, this reciprocity [deriving from Pride and Haartsen (1996)] is verified for different combinations of point sources and wave fields, because there are no significant differences for each pair of waves. We also verified the other two kinds of reciprocity for seismic waves generated by seismic point sources and electric waves generated by current sources. Thus we conclude that our program satisfies the reciprocity test.

As an example, we applied our numerical technique to a two-layer model with an explosion source near the free surface. For this model, a sand-sandstone interface is located at $100 \mathrm{~m}$ depth. Receivers are located at the free surface. The source time function adopted here is Ricker wavelet with a dominant frequency of $100 \mathrm{~Hz}$. We adopted the expression of Haartsen and Pride (1997) to calculate the moment $M_{0}$ generated by an explosion. The properties of the sand and sandstone layers are given in Table 1.
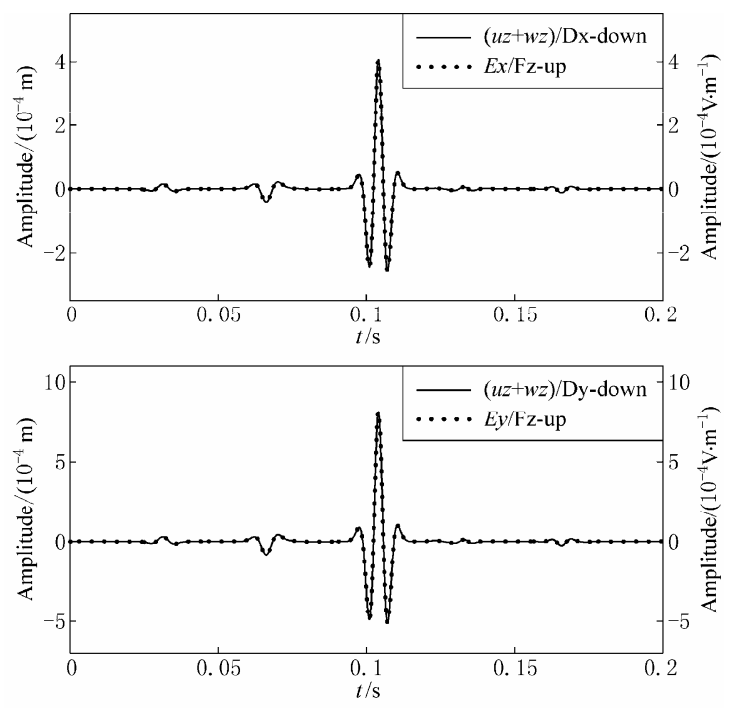

Figure 1 Reciprocity of coupled seismic and EM waves generated by different sources.

In order to show coupled waves clearly, we removed direct waves and free surface reflections, which are much more intense. Figure 2 shows none-zero field-component of displacement and EM waves at one receiver with a horizontal offset $50 \mathrm{~m}$ from the source. There are obvious electric signals accompanying $\mathrm{P}$ waves and magnetic signals accompanying $\mathrm{S}$ waves. Moreover, there are EM signals arriving earlier than $\mathrm{P}$ waves. They are activated when first $P$ wave arrives at the interface and travel at EM wave velocity which is much faster than seismic velocity. However, such P-to-EM conversion waves are very weak. In Figure 2, they are amplified by a factor of 500 to improve the visibility. Since the P-to-EM conversion waves are radiated from the interface, if they can be detected in the field, they may have potential application in the monitoring of oil/gas reservoirs, and the development of earthquake early warning system. 

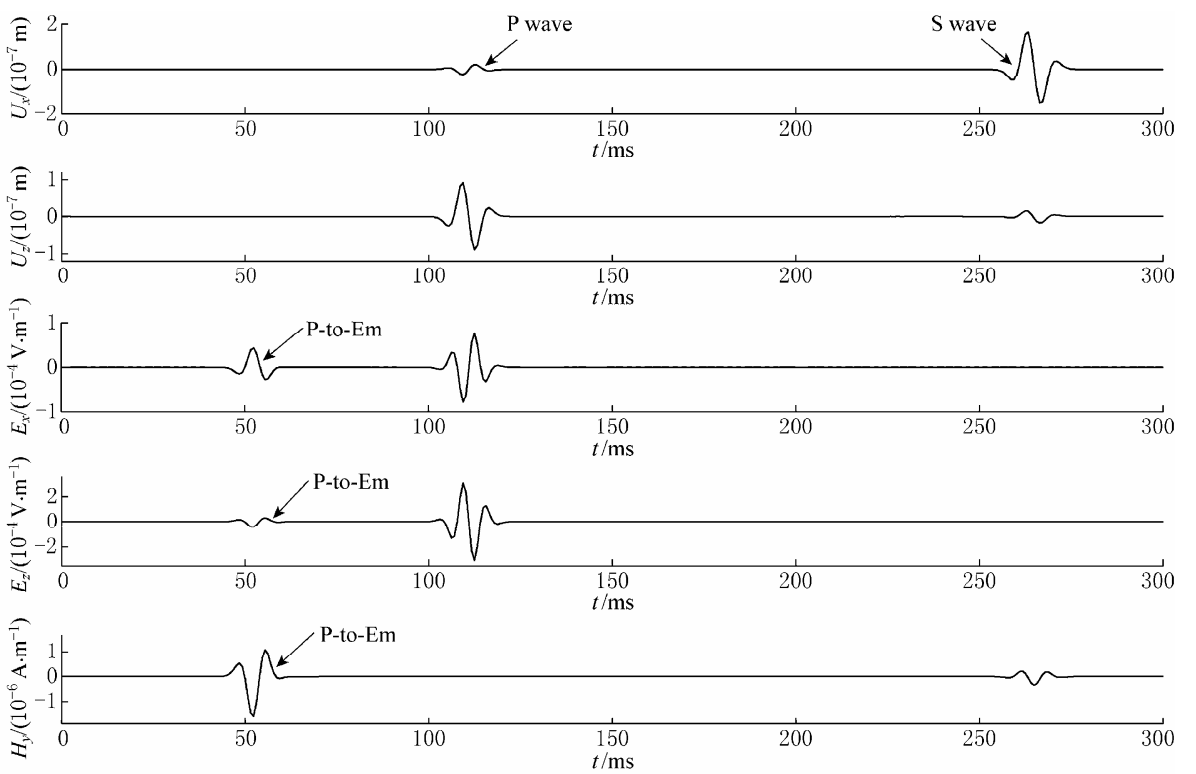

Figure 2 Seismograms and electroseismograms of the two-layer model with an explosion source near the free surface. A sand-sandstone interface is located at $100 \mathrm{~m}$ depth. The receiver is located at the free surface with a horizontal offset $50 \mathrm{~m}$ from the source. Direct waves and free surface reflections are omitted in order to show coupled waves clearly. The horizontal projection of source-receiver vector is in $x$-direction and $z$-direction is vertically downward. The P-to-EM conversions are amplified by a factor of 500 to improve the visibility.

\section{Conclusions}

Using a new straightforward evaluation of the generalized R/T coefficients, we have extended Chen's method of calculating synthetic seismogram to the numerical simulation of coupled seismic and EM waves in multi-layered porous media. We obtain the general solution of the full components of seismic and EM waves, including the vertical components of EM waves. After the reciprocity test, we conclude that the new numerical simulation technique developed here is reliable for investigating the coupling of seismic and EM waves in multi-layered porous media. We also perform a numerical example of a two-layer media with an explosion source. Besides EM signals accompanying with seismic waves, there are also weak P-to-EM conversion waves, which are radiated from the interface and travel at EM wave velocity. The P-to-EM conversion waves may have potential application in monitoring oil/gas reservoirs and early warning of earthquakes.

Acknowledgments This research is jointly supported by the Natural R\&D Special Fund for Public Welfare Industry (No.200808069) and National Natural Science Foundation of China (Nos.40974038, 40774028 and 40821062).

\section{References}

Aki K and Richards P G (1980). Quantitative Seismology: Theory and Methods. W. H. Freeman, San Francisco, CA.

Biot M A (1962). Mechanics of deformation and acoustic propagation in porous media. J Appl Phys 33: 1482-1 498.

Blau L W and Statham L (1936). Method and apparatus for seismic electric prospecting. US Patent No. 2054067.

Bolève A, Crespy A, Revil A, Janod F and Mattiuzzo J L (2007a). Streaming potentials of granular media: Influence of the Dukhin and Reynolds numbers. J Geophys Res 112, B08204, doi:10.1029/2006JB004673.

Bolève A, Revil A, Janod F, Mattiuzzo J L and Jardani A (2007b). Forward modeling and validation of a new formulation to compute self-potential signals associated with ground water flow. Hydrology and Earth System Sciences 11: 1661-1 671.

Bouchon M (1981). A simple method to calculate Green's functions for elastic layered media. Bull Seism Soc Amer 71: 959-971.

Bouchon M (2003). A review of the discrete wavenumber method. Pure Appl Geophys 160: 445-466.

Bouchon M and Aki K (1977). Discrete wave-number representation of seismic-source wave fields. Bull Seism Soc Amer 67: 259-277.

Chen X F (1993). A systematic and efficient method for computing seismic normal modes in layered half-space. Geophys J Int 115: 391-409.

Chen X F (1999). Seismogram synthesis in multi-layered half-space. Part I. Theoretical formulations. Earthquake Research in China 13: 149-174.

Chen X F (2007). Generation and propagation of seismic SH waves in multi-layered media with irregular interfaces. Advances in Geophysics $\mathbf{4 8}$ : 191-264.

Frenkel J (1944). On the theory of seismic and seismoelectric phenomena in a moist soil. J Phys 8: 230-241.

Garambois S and Dietrich M (2002). Full waveform numerical simulations of seismoelectromagnetic wave conversions in fluid-saturated stratified porous media. J Geophys Res 107, doi:10.1029/2001JB000316.

Ge Z X and Chen X F (2008). An efficient approach for simulating wave propagation with the boundary element method in multilayered media with 
irregular interfaces. Bull Seism Soc Amer 98, doi: 10.1785/0120080920.

Guan W and Hu H S (2008). Finite-difference modeling of electroseismic logging in a fluid saturated porous formation. J Comp Phys 228: 56335648.

Haartsen M W and Pride S R (1997). Electroseismic waves from point sources in layered media. J Geophys Res 102: 24 745-24 769.

Haines S S and Pride S R (2006). Seismoelectric numerical modeling on a grid. Geophysics 71: N57-N65.

Han Q and Wang Z (2001). Time-domain simulation of SH-wave-induced electromagnetic field in heterogeneous porous media: A fast finite-element algorithm. Geophysics 66: 448-461.

Hu H S, Guan W and Harris J (2007). Theoretical simulation of electroacoustic borehole logging in fluid-saturated porous formation. J Acoust Soc Am 122: $135-145$.

Hu H S, Liu J Q, Wang H B and Wang K X (2003). Simulation of acousto-electric well logging based on simplified Pride equations. Chinese $J$ Geophys 46: 259-264 (in Chinese with English abstract).

Huang Q (2002). One possible generation mechanism of co-seismic electric signals. Proc Japan Acad 78: 173-178.

Huang Q and Liu T (2006). Earthquakes and tide response of geoelectric potential field at the Niijima station. Chinese J Geophys 49: 1745-1 754 (in Chinese with English abstract).

Ivanov A G (1939). Effect of electrization of earth layers by elastic waves passing through them. Dokl Akad Nauk SSSR 24: 42-45.

Johnston M J S (1997). Review of electric and magnetic fields accompanying seismic and volcanic activity. Surv Geophys 18: 441-475.

Kennett B L N (1983). Seismic Wave Propagation in Stratified Media. Cambridge Univ Press, New York.

Luco J E and Apsel R J (1983). On the Green's function for a layered half-space: Part I. Bull Seism Soc Amer 73: 909-927.

Martin B E and Thomson C J (1997). Modelling surface waves in anisotropic structures II: Examples. Phys Earth Planet Inter 103: 253-279.

Martner S and Sparks N (1959). The electroseismic effect. Geophysics 24: 297-308.

Nagao T, Enomoto Y, Fujinawa Y, Hata M, Hayakawa M, Huang Q, Izutsu J, Kushida Y, Maeda K, Oike K, Uyeda S and Yoshino T (2002). Electro- magnetic anomalies associated with 1995 Kobe earthquake. J Geodyn 33: 401-411.

Park J (1996). Surface waves in layered anisotropic media. Geophys J Int 126: $173-183$.

Park S K, Johnston M J S, Madden T R, Morgan F D and Morrison H F (1993). Electromagnetic precursors to earthquakes in the ULF band: A review of observations and mechanisms. Rev Geophys 31: 117-132.

Pride S R (1994). Governing equations for the coupled electromagnetics and acoustics of porous media. Phys Rev B 50: 15 678-15 696.

Pride S R and Haartsen M W (1996). Electroseismic wave properties. $J$ Acoust Soc Am 100: 1301-1315.

Revil A and Leroy P (2004). Governing equations for ionic transport in porous shales. J Geophys Res 109, B03208, doi:10.1029/2006JB002755.

Revil A and Linde N (2006). Chemico-electromechanical coupling in microporous media. J Coll Interf Sci 302: 682-694.

Revil A, Naudet V, Nouzaret J and Pessel M (2003). Principles of electrography applied to self-potential electrokinetic sources and hydrogeological applicatons. Water Resources Res 39: 1 114, doi: 10.1029/2001WR000916.

Thompson R R (1936). The seismic electric effect. Geophysics 1: 327-335.

Thompson A H and Gist G A (1993). Geophysical applications of electrokinetic conversion. The Leading Edge 12: 1 169-1 173.

Thompson A H, Hornbostel S, Burns J, Murray T, Raschke R, Wride J, McCammon P, Sumner J, Haake G, Bixby M, Ross W, White B S, Zhou M and Peczak P (2007). Field tests of electroseismic hydrocarbon detection. Geophysics 72: N1-N9.

Zhu Z and Toksöz M N (1999). Seismoelectric and seismomagnetic measurements in fractured borehole models. The 69th Annual International Meeting, SEG, Expanded Abstracts, 144-147.

Zhu Z and Toksöz M N (2003). Crosshole seismoelectric measurements in borehole models with fractures. Geophysics 68: 1 519-1 524.

Zhu Z, Haartsen M W and Toksöz M N (2000). Experimental studies of seismoelectric conversions in fluid-saturated porous media. $J$ Geophys Res 105: 28 055-28 064 .

\section{Appendix A: Scalar and vector wave-function basis}

For scalar function, we take cylindrical harmonic function $Y_{k}^{m}(r, \theta)$ as basis function with which an arbitrary scalar function $F(r, \theta, z)$ can be expanded as follows

$$
F(r, \theta, z)=\frac{1}{2 \pi} \sum_{m=-\infty}^{+\infty} \int_{0}^{+\infty} f_{m}(k, z) Y_{k}^{m}(r, \theta) k \mathrm{~d} k,
$$

where, $f_{m}(k, z)$ is the expansion coefficient of $F(r, \theta, z)$, and the function basis $Y_{k}^{m}(r, \theta)$ has following explicit form

$$
Y_{k}^{m}(r, \theta)=J_{m}(k r) e^{i m \theta} \text { for } m=0, \pm 1, \pm 2, \cdots ;
$$

where $J_{m}(k r)$ is a Bessel function of order $m$.

For vector function, we take the following set of vector functions as basis,

$$
\left\{\begin{array}{l}
\boldsymbol{T}_{k}^{m}(r, \theta)=k^{-1} \nabla \times\left[\hat{\boldsymbol{e}}_{\mathrm{z}} Y_{k}^{m}(r, \theta)\right] \\
\boldsymbol{S}_{k}^{m}(r, \theta)=k^{-1} \nabla Y_{k}^{m}(r, \theta) \\
\boldsymbol{R}_{k}^{m}(r, \theta)=-\hat{\boldsymbol{e}}_{z} Y_{k}^{m}(r, \theta)
\end{array}\right.
$$

It had been proven that this set of vector basis function is orthogonal (e.g. Aki and Richards, 1980) and complete (Chen, 1999). Therefore, an arbitrary vector function, say $\boldsymbol{A}(r, \theta, z, \omega)$, can be expanded with the above vector basis function as

$$
\begin{gathered}
\boldsymbol{A}(r, \theta, z, \omega)=\frac{1}{2 \pi} \sum_{m=-\infty}^{+\infty} \int_{0}^{+\infty}\left\{A_{T, m}(z, k, \omega) \boldsymbol{T}_{k}^{m}(r, \theta)+\right. \\
\left.A_{S, m}(z, k, \omega) \boldsymbol{S}_{k}^{m}(r, \theta)+A_{R, m}(z, k, \omega) \boldsymbol{R}_{k}^{m}(r, \theta)\right\} k \mathrm{~d} k,
\end{gathered}
$$

where $A_{T, m}, A_{S, m}$ and $A_{R, m}$ are the expansion coefficients of $\boldsymbol{A}$ under the vector function basis $\left\{\boldsymbol{T}_{k}^{m}(r, \theta), \boldsymbol{S}_{k}^{m}(r, \theta)\right.$, $\left.\boldsymbol{R}_{k}^{m}(r, \theta)\right\}$ given by equation (10). If the vector function $\boldsymbol{A}$ is known, these expansion coefficients can be evaluated by the following formulae,

$$
A_{T, m}(z, k, \omega)=\int_{0}^{2 \pi+\infty} \int_{0}^{+\infty} A(r, \theta, z, \omega) \mathrm{g}\left[\mathbf{T}_{k}^{m}(r, \theta)\right]^{*} r \mathrm{~d} r \mathrm{~d} \theta,
$$


$A_{S, m}(z, k, \omega)=\int_{0}^{2 \pi} \int_{0}^{+\infty} \boldsymbol{A}(r, \theta, z, \omega) \mathrm{g}\left[\boldsymbol{S}_{k}^{m}(r, \theta)\right]^{*} r \mathrm{~d} r \mathrm{~d} \theta, \quad(\mathrm{A}-6)$

where, the symbol * indicates the complex conjugate.

$A_{R, m}(z, k, \omega)=\int_{0}^{2 \pi} \int_{0}^{+\infty} A(r, \theta, z, \omega) \mathrm{g}\left[\boldsymbol{R}_{k}^{m}(r, \theta)\right]^{*} r \mathrm{~d} r \mathrm{~d} \theta, \quad(\mathrm{A}-7)$

\section{Appendix B: Expressions of $\boldsymbol{A}$ and $\boldsymbol{F}$}

$$
\begin{aligned}
& \boldsymbol{A}^{\mathrm{SHTE}}=\left[\begin{array}{cccc}
0 & G^{-1} & 0 & 0 \\
G k^{2}-\rho \omega^{2}-\mathrm{i} \frac{\kappa}{\eta} \rho_{\mathrm{f}}^{2} \omega^{3} & 0 & 0 & -\mathrm{i} L \rho_{\mathrm{f}} \omega \\
-L \rho_{\mathrm{f}} \omega^{2} & 0 & 0 & -\mathrm{i} \frac{k^{2}}{\mu \omega}-\sigma+\mathrm{i} \varepsilon \omega \\
0 & 0 & \mathrm{i} \mu \omega & 0
\end{array}\right], \\
& \boldsymbol{F}^{\mathrm{SHTE}}(z)=\left[\begin{array}{l}
\boldsymbol{F}_{1}^{\mathrm{SHTE}}(z) \\
\boldsymbol{F}_{2}^{\mathrm{SHTE}}(z)
\end{array}\right], \\
& \boldsymbol{F}_{1}^{\mathrm{SHTE}}(z)=\left[0, \quad-F_{T, m}(z, k, \omega)-\mathrm{i} \frac{\kappa}{\eta} \rho_{\mathrm{f}} \omega f_{T, m}(z, k, \omega)\right]^{\mathrm{T}}, \\
& \boldsymbol{F}_{2}^{\mathrm{SHTE}}(z)=\left[-L f_{T, m}(z, k, \omega)+\mathrm{i} \frac{k}{\mu \omega} M_{R, m}(z, k, \omega)-C_{T, m}(z, k, \omega), \quad-M_{S, m}(z, k, \omega)\right]^{\mathrm{T}},
\end{aligned}
$$

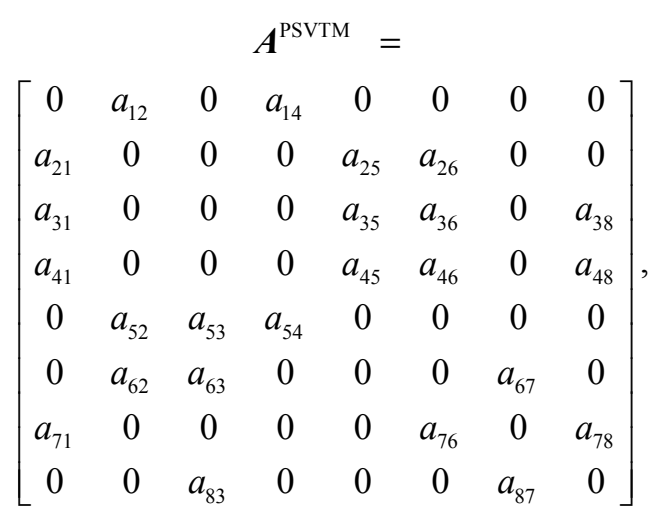

$$
\begin{aligned}
& \boldsymbol{F}_{2}^{\mathrm{PSVTM}}(z)=\left[\begin{array}{c}
-F_{R, m}(z, k, \omega) \\
-f_{R, m}(z, k, \omega)+L \chi C_{R, m}(z, k, \omega) \\
L f_{s, m}(z, k, \omega)+C_{s, m}(z, k, \omega) \\
\chi \frac{\kappa}{\eta} k C_{R, m}(z, k, \omega)+M_{T, m}(z, k, \omega)
\end{array}\right] \\
& a_{12}=k, a_{14}=G^{-1}, a_{21}=\frac{\left(C^{2}-\lambda M\right) k}{\Delta}, \\
& a_{25}=\frac{M}{\Delta}, a_{26}=-\frac{C}{\Delta} ; \\
& \boldsymbol{F}^{\text {PSVTM }}(z)=\left[\begin{array}{l}
\boldsymbol{F}_{1}^{\text {PSVTM }}(z) \\
\boldsymbol{F}_{2}^{\text {PSVTM }}(z)
\end{array}\right],
\end{aligned}
$$

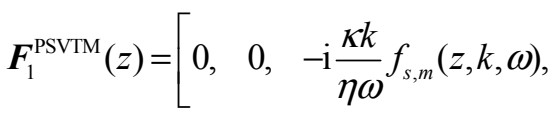

$$
\begin{aligned}
& \left.-F_{s, m}(z, k, \omega)-\mathrm{i} \frac{\kappa}{\eta} \rho_{\mathrm{f}} \omega f_{s, m}(z, k, \omega)\right]^{\mathrm{T}}, \\
& a_{31}=-k\left(\frac{2 G C}{\Delta}+\mathrm{i} \frac{\kappa}{\eta} \rho_{\mathrm{f}} \omega\right), a_{35}=-\frac{C}{\Delta}, \\
& a_{36}=\frac{1}{M}+\frac{C^{2}}{\Delta M}+\mathrm{i} \frac{\kappa k^{2}}{\eta \omega}, a_{38}=-\mathrm{i} \frac{L k}{\omega} ; \\
& a_{41}=\left(1-\frac{G M}{\Delta}\right) 4 G k^{2}-\rho \omega^{2}-\mathrm{i} \frac{\kappa}{\eta} \rho_{\mathrm{f}}^{2} \omega^{3}, \\
& a_{45}=k\left(1-\frac{2 G M}{\Delta}\right) \text {; }
\end{aligned}
$$




$$
\begin{gathered}
a_{46}=\frac{2 G C k}{\Delta}+\mathrm{i} \frac{\kappa}{\eta} \rho_{\mathrm{f}} \omega k, a_{48}=-\mathrm{i} L \rho_{\mathrm{f}} \omega ; \\
a_{52}=-\rho \omega^{2}, a_{53}=-\rho_{\mathrm{f}} \omega^{2}, a_{54}=-k ; \\
a_{62}=-\rho_{\mathrm{f}} \omega^{2}, a_{63}=-\mathrm{i}(\sigma-\mathrm{i} \varepsilon \omega) \chi \omega ; \\
a_{67}=L k \chi ; \\
a_{71}=L \rho_{\mathrm{f}} \omega^{2}, a_{76}=-L k, a_{78}=\sigma-\mathrm{i} \varepsilon \omega ; \\
a_{83}=-\mathrm{i} L \chi \omega k, a_{87}=\frac{\kappa}{\eta} \chi k^{2}-\mathrm{i} \mu \omega ;
\end{gathered}
$$

where,

\section{Appendix C: Determination of $\boldsymbol{R}_{u d}^{(0)}$ and $\boldsymbol{R}_{d u}^{(N+1)}$}

In bottom layer, there are no up-going waves i.e., $\boldsymbol{a}_{\mathrm{u}}^{(N+1)}=0$. We obtain $\boldsymbol{R}_{\mathrm{du}}^{(N+1)}=0$. If the top layer is a half-space, we have equation $\boldsymbol{R}_{\mathrm{ud}}^{(0)}=0$; if the top layer has a free surface, we have $\boldsymbol{a}_{\mathrm{d}}^{(1)}=\hat{\boldsymbol{R}}_{\mathrm{ud}}^{(0)}\left[\boldsymbol{a}_{\mathrm{u}}^{(1)}+\right.$ $\delta_{1, s} \boldsymbol{b}_{\mathrm{u}}\left(z^{(0)}\right)$ ], and two extra boundary conditions. One is the traction-free condition, $\tau_{T, m}=0, \quad \tau_{S, m}=0, \tau_{R, m}=0$, $\widetilde{P}=0$, for $z=z^{(0)}$. The other is the continuity of the horizontal components of the electric and magnetic fields (see Pride and Haartsen, 1996) at the free surface.

$$
\begin{gathered}
\lambda=K_{G}-\frac{2}{3} G, \Delta=(\lambda+2 G) M-C^{2}, \\
\chi=\left[\frac{\kappa}{\eta}(\sigma-\mathrm{i} \varepsilon \omega)-L^{2}\right]^{-1} ;
\end{gathered}
$$

and $k$ is the horizontal wave-number; $F_{T, m}, F_{S, m}, F_{R, m}$, $f_{T, m}, f_{S, m}, f_{R, m}, M_{T, m}, M_{S, m}, M_{R, m}, C_{T, m}, C_{S, m}$, and $C_{R, m}$ are the expansion coefficients of those known mechanical, magnetic and electric source vectors $\boldsymbol{F}, \boldsymbol{f}, \boldsymbol{M}$ and $\boldsymbol{C}$, respectively.
Finally, we obtain that, for SHTE model,

$$
\begin{gathered}
\boldsymbol{R}_{\mathrm{ud}}^{(0)}=\left[\begin{array}{cc}
\Theta_{21}^{(1)} & \Theta_{22}^{(1)} \\
\mu_{0} \Theta_{31}^{(1)}-q_{0} \Theta_{41}^{(1)} & \mu_{0} \Theta_{32}^{(1)}-q_{0} \Theta_{42}^{(1)}
\end{array}\right]^{-1} \\
{\left[\begin{array}{cc}
-\Theta_{23}^{(1)} & -\Theta_{24}^{(1)} \\
-\mu_{0} \Theta_{33}^{(1)}+q_{0} \Theta_{43}^{(1)} & -\mu_{0} \Theta_{34}^{(1)}+q_{0} \Theta_{44}^{(1)}
\end{array}\right] \Lambda_{u}^{(1)}\left(z^{(0)}\right)} \\
q_{0}^{2}+p^{2}=\mu_{0} \varepsilon_{0}, \quad p=k / \omega, \quad \operatorname{Im}\left\{q_{0}\right\}>0,
\end{gathered}
$$

and for PSVTM model,

$$
\boldsymbol{R}_{\mathrm{ud}}^{(0)}=-\boldsymbol{\Gamma} \boldsymbol{\Xi} \boldsymbol{\Lambda}_{\mathrm{u}}^{(1)}\left(z^{(0)}\right),
$$

$$
\begin{aligned}
& \boldsymbol{\Gamma}=\left[\begin{array}{cccc}
\Theta_{41}^{(1)} & \Theta_{42}^{(1)} & \Theta_{43}^{(1)} & \Theta_{44}^{(1)} \\
\Theta_{51}^{(1)} & \Theta_{52}^{(1)} & \Theta_{53}^{(1)} & \Theta_{54}^{(1)} \\
\Theta_{61}^{(1)} & \Theta_{62}^{(1)} & \Theta_{63}^{(1)} & \Theta_{64}^{(1)} \\
q_{0} \Theta_{71}^{(1)}+\varepsilon_{0} \Theta_{81}^{(1)} & q_{0} \Theta_{72}^{(1)}+\varepsilon_{0} \Theta_{82}^{(1)} & q_{0} \Theta_{73}^{(1)}+\varepsilon_{0} \Theta_{83}^{(1)} & q_{0} \Theta_{74}^{(1)}+\varepsilon_{0} \Theta_{84}^{(1)}
\end{array}\right], \\
& \boldsymbol{\Xi}=\left[\begin{array}{cccc}
\Theta_{45}^{(1)} & \Theta_{46}^{(1)} & \Theta_{47}^{(1)} & \Theta_{48}^{(1)} \\
\Theta_{55}^{(1)} & \Theta_{56}^{(1)} & \Theta_{57}^{(1)} & \Theta_{58}^{(1)} \\
\Theta_{65}^{(1)} & \Theta_{66}^{(1)} & \Theta_{67}^{(1)} & \Theta_{68}^{(1)} \\
q_{0} \Theta_{75}^{(1)}+\varepsilon_{0} \Theta_{85}^{(1)} & q_{0} \Theta_{76}^{(1)}+\varepsilon_{0} \Theta_{86}^{(1)} & q_{0} \Theta_{77}^{(1)}+\varepsilon_{0} \Theta_{87}^{(1)} & q_{0} \Theta_{78}^{(1)}+\varepsilon_{0} \Theta_{88}^{(1)}
\end{array}\right], \\
& q_{0}{ }^{2}+p^{2}=\mu_{0} \varepsilon_{0} \quad\left(p=k / \omega, \quad \operatorname{Im}\left\{q_{0}\right\}>0\right),
\end{aligned}
$$

where $\Theta_{i j}^{(1)}$ are the elements of the matrix $\boldsymbol{\Theta}^{(1)}$. 